\title{
Random regular graphs of non-constant degree: connectivity and Hamiltonicity
}

\author{
Colin Cooper* $\quad$ Alan Frieze ${ }^{\dagger} \quad$ Bruce Reed $^{\ddagger}$
}

September 9, 2001

\begin{abstract}
Let $G_{r}$ denote a graph chosen uniformly at random from the set of $r$-regular graphs with vertex set $\{1,2, \ldots, n\}$ where $3 \leq r \leq c_{0} n$ for some small constant $c_{0}$. We prove that with probability tending to 1 as $n \rightarrow \infty, G_{r}$ is $r$-connected and Hamiltonian.
\end{abstract}

\section{Introduction}

The properties of random $r$-regular graphs have received much attention. For a comprehensive discussion of this topic, see the recent survey by Wormald [22] or Chapter 9 of the book, Random Graphs, by Janson, Łuczak and Ruciński [12].

A major obstacle in the development of the subject has been a lack of suitable techniques for modelling simple random graphs over the entire range, $0 \leq r \leq n-1$, of possible values of $r$. The classical method for generating uniformly distributed simple $r$-regular graphs, is by rejection sampling using the configuration model of Bollobás [3]. The configuration model is a probabilistic interpretation of a counting formula of Bender and Canfield [2]. The method is most easily applied when $r$ is constant or grows slowly with $n$, the number of vertices, as $n$ tends to infinity. The formative paper [3] on this topic considered the case where $r=O\left((\log n)^{1 / 2}\right)$. McKay [16] and McKay

*Department of Mathematical and Computing Sciences, Goldsmiths College, University of London, New Cross, London SE14 6NW. Research supported by the STORM Research Centre, UNL. E-mail: c.cooper@gold.ac.uk.

$\dagger$ Department of Mathematical Sciences, Carnegie Mellon University, Pittsburgh PA15213, U.S.A., Supported in part by NSF grant CCR-9818411. E-mail: alan@random.math. cmu.edu.

$\ddagger$ Equipe Combinatoire, CNRS, Univ. de Paris VI, 4 Place Jussieu, Paris 75005, France. E-mail: reed@ecp6.jussieu.fr. 
and Wormald $[17,18]$ subsequently gave alternative approaches which are useful for $r=o\left(n^{1 / 2}\right)$ or $r=\Omega(n)$.

We use edge switching techniques extensively in this paper and note that these techniques have been successfully applied in a number of places e.g. [16], [17, 18], [9], [14] and [13].

Let $G_{r}$ denote a graph chosen uniformly at random from the set $\mathcal{G}_{r}$ of simple $r$-regular graphs with vertex set $V=\{1,2, \ldots, n\}$. We consider properties of simple $r$-regular graphs for the case where $r \rightarrow \infty$ as $n \rightarrow \infty$, but $r=o(n)$. The properties we study are vertex $r$-connectivity and Hamiltonicity. These properties are also studied, in a recent paper by Krivelevich, Sudakov, $\mathrm{Vu}$ and Wormald [13], for the case where $r(n) \geq \sqrt{n} \log n$. Our paper complements [13] both in both in the range of $r$ studied and in the techniques applied.

Theorem 1 Assume $3 \leq r \leq c_{0}$ for some small positive absolute constant $c_{0}$. Then with probability tending to 1 as $n \rightarrow \infty$,

(a) $G_{r}$ is r-connected.

(b) $G_{r}$ is Hamiltonian.

The results of Theorem 1 are well known for $r$ constant. Result (a) is from Bollobás [4] and (b) is from Robinson and Wormald [20, 21], Bollobás [5], Fenner and Frieze [8]. For $r=o\left(n^{1 / 2}\right)$ such results could have been proved with the help of the models of [16] and [17]. In fact this was done, for Hamiltonicity, up to $r=o\left(n^{1 / 5}\right)$, in an unpublished work by Frieze [9], and for $r$-connectivity, up to $r \leq n^{.002}$ by Luczak [15].

As [13] proves the case where $r \geq n^{1 / 2} \log n$, this implies $G_{r}$ is $r$-connected and Hamiltonian $\mathbf{w h p}^{1}$ for all $3 \leq r \leq n-4$.

\section{Generating graphs with a fixed degree sequence.}

Let $\mathbf{d}=\left(d_{1}, d_{2}, \ldots, d_{n}\right)$, and let $2 D=\left(d_{1}+d_{2}+\cdots+d_{n}\right)$. Let $\mathcal{G}_{\mathbf{d}}$ be the set of simple graphs $G$ with vertex set $V=[n]$, degree sequence $\mathbf{d}$, and $D$ edges.

Let $\Omega$ be the set of all $(2 D) ! /\left(D ! 2^{D}\right)$ partitions of $W=[2 D]$ into $D$ 2-element sets. An element of $\Omega$ is a configuration. The constituent 2-element sets of a configuration $F$ are referred to as the edges of $F$.

Let $W_{1}, W_{2}, \ldots, W_{n}$ be the natural ordered partition $P_{\mathbf{d}}$ of $W=[2 D]$ into sets of size $\left|W_{i}\right|=d_{i}$, and where $\left(\max W_{i}\right)+1=\min W_{i+1}$ for $i<n$.

\footnotetext{
${ }^{1} \mathrm{~A}$ sequence of events $\mathcal{E}_{n}$ is said to occur with high probability $(\mathbf{w h p})$ if $\lim _{n \rightarrow \infty} \operatorname{Pr}\left(\mathcal{E}_{n}\right)=1$.
} 
Let $\Omega_{\mathbf{d}}$ be $\Omega$ with the understanding that the underlying set $W$ is partitioned into $P_{d}$. The degree sequence of an element $F$ of $\Omega_{\mathbf{d}}$ is $\mathbf{d}$. We often write $\Omega$ for $\Omega_{\mathbf{d}}$ when the context is clear. Define $\phi_{P_{\mathbf{d}}}: W \rightarrow[n]$ by $\phi(w)=i$ if $w \in W_{i}$. Let $\gamma(F)$ denote the multigraph with vertex set $[n]$ and edge multiset $E_{F}=\{\{\phi(x), \phi(y)\}:\{x, y\} \in F\}$.

Definition: Let $\Omega_{\mathbf{d}}^{*}$ denote those configurations $F$ for which $\gamma(F)$ is simple relative to $P_{\mathbf{d}}$.

Remark 1 Note that each member of $\mathcal{G}_{\mathbf{d}}$ is the image under $\gamma$ of precisely $\prod_{i=1}^{n} d_{i}$ ! members of $\Omega_{\mathbf{d}}^{*}$. Thus sampling $F$ uniformly from $\Omega_{\mathbf{d}}^{*}$ induces the uniform measure on $\gamma(F)$ and is equivalent to sampling uniformly from $\mathcal{G}_{\mathbf{d}}$.

If $d_{i}=r,(1 \leq i \leq n)$ we will say the configuration, $F$, is $r$-regular. The probability $\left|\Omega^{*}\right| /|\Omega|$ that the underlying $r$-regular multigraph $\gamma(F)$ of such a configuration $F$ is simple is $\exp \left(-\Theta\left(r^{2}\right)\right)$. For $r=o\left(n^{1 / 2}\right)$ this follows from [17, 18] and for larger values of $r$ from Lemma 2 below. This result allows us to prove many results directly via configurations and then condition the probability estimates for simple graphs.

Lemma 1 Let $\Delta=\max _{i \in[n]} d_{i}$. Suppose that $\Delta \leq n / 1000$ and that $\mathbf{d}$ satisfies $\min _{i \in[n]} d_{i} \geq \Delta / 4$. Given $a, b \in[n]$, if $G$ is sampled u.a.r. from $\mathcal{G}_{\mathbf{d}}$, then

$$
\operatorname{Pr}(\{a, b\} \in E(G)) \leq \frac{20 \Delta}{n} .
$$

\section{Proof Let}

$$
\Omega_{1}=\left\{G \in \mathcal{G}_{\mathbf{d}}:\{a, b\} \in E(G)\right\} \text { and } \Omega_{2}=\mathcal{G}_{\mathbf{d}} \backslash \Omega_{1} \text {. }
$$

We consider the set $X$ of pairs $\left(G_{1}, G_{2}\right) \in \Omega_{1} \times \Omega_{2}$ such that $G_{2}$ is obtained from $G_{1}$ by deleting disjoint edges $\{a, b\},\left\{x_{1}, y_{1}\right\},\left\{x_{2}, y_{2}\right\}$ and replacing them by $\left\{a, x_{1}\right\},\left\{y_{1}, y_{2}\right\}$, $\left\{b, x_{2}\right\}$. Given $G_{1}$, we can choose $\left\{x_{1}, y_{1}\right\},\left\{x_{2}, y_{2}\right\}$ to be any ordered pair of disjoint edges which are not incident with $a, b$ or their neighbours and such that $\left\{y_{1}, y_{2}\right\}$ is not an edge of $G_{1}$. Thus each $G_{1} \in \Omega_{1}$ is in at least $\left(D-\left(2 \Delta^{2}+1\right)\right)\left(D-\left(4 \Delta^{2}+2\right)\right)$ pairs. Each $G_{2} \in \Omega_{2}$ is in at most $2 D \Delta^{2}$ pairs. The factor of 2 arises because a suitable edge $\left\{y_{1}, y_{2}\right\}$ of $G_{2}$ has an orientation relative to the switching back to $G_{1}$. As $D \geq n \Delta / 8$ it follows that

$$
\frac{\left|\Omega_{1}\right|}{\left|\Omega_{2}\right|} \leq \frac{2 D \Delta^{2}}{\left(D-\left(2 \Delta^{2}+1\right)\right)\left(D-\left(4 \Delta^{2}+2\right)\right)} \leq \frac{20 \Delta}{n} .
$$

Lemma 2 Suppose $100 \leq r \leq n / 1000$. Let $d_{j}=r, 1 \leq j \leq n$. If $F$ is chosen uniformly at random (u.a.r) from $\Omega$ then for $n$ sufficiently large,

$$
\operatorname{Pr}\left(F \in \Omega^{*}\right) \geq e^{-2 r^{2}}
$$


Proof Consider the following algorithm from Frieze and Luczak [11]:

\section{Algorithm Generate}

\section{begin}

$D:=r n / 2$

$F_{0}:=\emptyset$

Let $\boldsymbol{\sigma}=\left(x_{1}, x_{2}, \ldots, x_{2 D-1}, x_{2 D}\right)$ be an ordering of $W$

For $i=1$ to $D$ do

begin

$$
F_{i}:= \begin{cases}F_{i-1} \cup\left\{\left\{x_{2 i-1}, x_{2 i}\right\}\right\} & \text { (With probability } \left.\frac{1}{2 i-1}\right) \mathbf{A} \\ F_{i-1} \cup\left\{\left\{x_{2 i-1}, z_{1}\right\},\left\{x_{2 i}, z_{2}\right\}\right\}-\left\{z_{1}, z_{2}\right\} & \text { (With probability } \left.\frac{2 i-2}{2 i-1}\right) \mathbf{B}\end{cases}
$$

Here $\left\{z_{1}, z_{2}\right\}$ is chosen u.a.r from $F_{i-1}$ and $z_{1}$ is chosen u.a.r from $\left\{z_{1}, z_{2}\right\}$.

\section{end}

Output $F:=F_{D}$

\section{end}

We first prove that GENERATE produces a u.a.r member of $\Omega$ whatever the ordering $\boldsymbol{\sigma}=\left(x_{1}, x_{2}, \ldots, x_{2 D}\right)$ of $W$. We then describe an ordering $\boldsymbol{\sigma}$ from which we can prove the lemma.

Let $W^{(i)}=\left(x_{1}, x_{2}, \ldots, x_{2 i}\right)$ and let $\Omega_{i}$ be the set of configurations of $W^{(i)}$. We show inductively that $F_{i}$ is a random member of $\Omega_{i}$. This clearly true for $i=1$ and so assume that for some $i \geq 2$ we have that $F_{i-1}$ is chosen u.a.r from $\Omega_{i-1}$.

Now consider a bipartite graph $H$ with vertex bipartition $\left(\Omega_{i-1}, \Omega_{i}\right)$ and an edge $\left(F, F^{\prime}\right)$ whenever $F^{\prime}=F \cup\left\{x_{2 i-1}, x_{2 i}\right\}$ or $F^{\prime}=(F \backslash\{a, b\}) \cup\left\{\left\{a, x_{2 i-1}\right\},\left\{b, x_{2 i}\right\}\right\}$ for some $\{a, b\} \in F$. Each $F \in \Omega_{i-1}$ has degree $2 i-1$ in $H$ and each $F^{\prime} \in \Omega_{i}$ has degree 1. Our algorithm chooses $F$ uniformly from $\Omega_{i-1}$ (induction) and then uniformly chooses an $H$-edge leaving $F$. This implies uniformity in $\Omega_{i}$.

Label the configuration points in set $W_{k}$ of the partition, as $\{(k-1) r+j: 1 \leq j \leq r\}$. For the ordering $\boldsymbol{\sigma}$ of $W$, we specify that $x_{i}$ is always chosen as one of the remaining points for which $\phi\left(x_{i}\right)$ occurs as little as possible in the sequence $\left(\phi\left(x_{1}\right), \ldots, \phi\left(x_{i-1}\right)\right)$. To be specific, when $i=(j-1) n+k,(1 \leq k \leq n, 1 \leq j \leq r)$, define $x_{i}$ to be the point in $W_{k}$ with label $(k-1) r+j$.

Let $\Omega_{i}^{*}=\left\{F \in \Omega_{i}: \gamma(F)\right.$ is simple $\}$. Let $\Delta_{i}=\lceil 2 i / n\rceil$ denote the maximum degree in $\gamma\left(F_{i}\right)$. Let the edge $\left\{\phi\left(x_{2 i-1}\right), \phi\left(x_{2 i}\right)\right\}=\{a, b\}$ and let $\left\{\phi\left(z_{1}\right), \phi\left(z_{2}\right)\right\}=\{c, d\}$. We will prove that

$$
\operatorname{Pr}\left(F_{i} \in \Omega_{i}^{*} \mid F_{i-1} \in \Omega_{i-1}^{*}\right) \geq \begin{cases}1 & 2 i \leq n \\ \left(1-\frac{60 \Delta_{i}}{(2 i-1) n}-\frac{2 \Delta_{i}^{2}+2 \Delta_{i}}{i-1}\right) & n<2 i \leq r n .\end{cases}
$$


If $i \leq n / 2$ then $F_{i}$ induces a matching. If $i>n / 2$ and if at the $i$ th step of GENERATE, $\{a, b\}$ already exists in Case A or is equal to $\{c, d\}$ in Case B then $F_{i}$ will not be simple. The probability the edge $\{a, b\}$ exists, in the corresponding simple random graph, is at most $\frac{20 \Delta_{i}}{n}$, by Lemma 1 . Thus the probability the edge exists (Case A) or exists and is selected (Case B) is at most

$$
\frac{20 \Delta_{i}}{n}\left(\frac{1}{2 i-1}+\frac{2 i-2}{2 i-1} \frac{1}{i-1}\right)=\frac{60 \Delta_{i}}{(2 i-1) n} .
$$

Assume now that the $i$ th step is type B and $\{a, b\} \neq\{c, d\}$.

When $\{a, b\} \cap\{c, d\} \neq \emptyset$, a loop may be created. This happens with probability at most $2 \Delta_{i} /(i-1)$.

When one of $a, b$ is adjacent to $c$ or $d$, a parallel edge may be created. This happens with probability at most $2 \Delta_{i}^{2} /(i-1)$.

All cases have been covered and the result follows from iterating (1) for $i \leq r n / 2$.

Remark 2 In Lemma 7 we need to run algorithm GENERATE starting with a configuration $F_{0}$ on $\left[2 D^{\prime}\right]$ and and restricting our random choice of $\left\{z_{1}, z_{2}\right\}$ to $F \backslash F_{0}$. The output is then $F_{0}$ plus a random configuration on $W=\left[2 D^{\prime}+1,2 D^{\prime}+2 D\right]$.

At this point we describe a simpler algorithm CONSTRUCT for obtaining a u.a.r configuration.

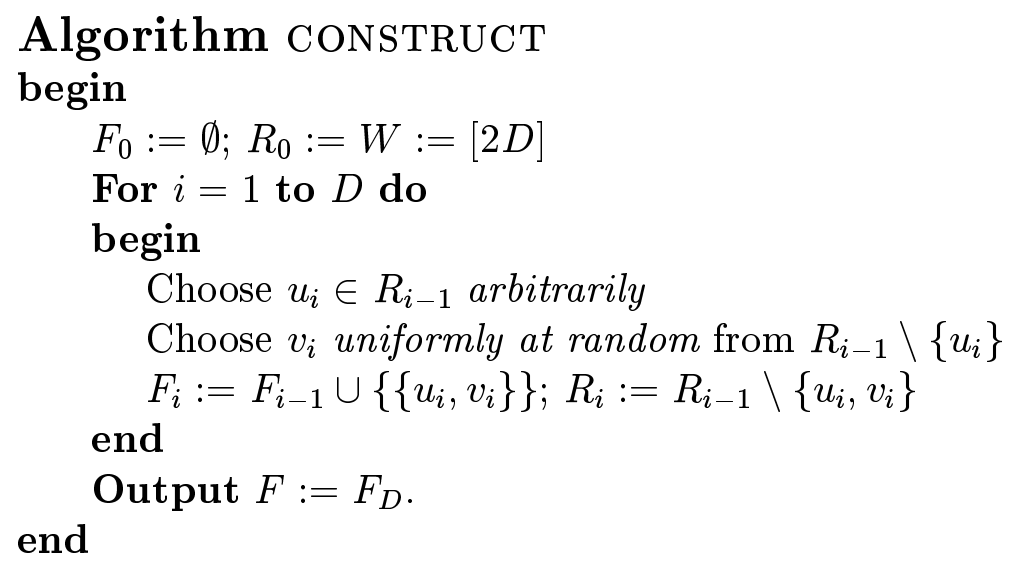

Remark 3 Neither of the algorithms generating $F_{D}$ use any information about the partition $P_{\mathbf{d}}$ associated with the configuration. After iteration $i, F_{i}$ is a u.a.r element of $\Omega_{i}$. We can, if we wish, complete a certain number $I$ of iterations using CONSTRUCT and then switch to GENERATE. Instead of initializing the ordering $\boldsymbol{\sigma}$ used in algorithm GENERATE with $W$ we initialize $\boldsymbol{\sigma}$ with $R_{I}$, the remaining unmatched points. 


\section{3 r-Connectivity}

We now prove Theorem 1(a). Since the result is already known for $r$ constant, we can assume that $10^{6} \leq r \leq c_{0} n$, where $c_{0}$ is sufficiently small.

For a simple graph $G$ with edge set $E$, the disjoint neighbour set, $N(S)$, of a set of vertices $S$ is defined as $N(S)=\{w \notin S: \exists v \in S$ s.t. $\{v, w\} \in E\}$. When $S$ is a singleton $\{v\}$ we use the notation $N(v)$.

Lemma 3 Let $\mathcal{Q}_{1} \subseteq \mathcal{G}_{r}$ be the event that for all vertices $v, w \in V$ of $G_{r}$ :

(a) If $r=o(n)$ then $|N(v) \cap N(w)| \leq 10+o(r)$.

(b) If $\log ^{2} n \leq r \leq n$ then $|N(v) \cap N(w)| \leq r^{2} / n+5 \sqrt{r \log n}$.

Then $\operatorname{Pr}\left(\overline{\mathcal{Q}_{1}}\right)=O\left(1 / n^{2}\right)$.

Proof Throughout this proof, we fix a vertex $v$ and the set $S=N(v)$, of vertices which are the (disjoint) neighbours of $v$. Let $w$ be a fixed vertex of $V-v$.

Let $\mathcal{F}(S)=\left\{G: G=G_{r}-v, N(v)=S\right\}$ be the set of graphs $G$ with vertex set $V-v$ formed by deleting $v$ from those $r$-regular graphs, $G_{r}$, for which $N(v)=S$. Thus $|S|=r$, and the vertices in $S$ have degree $r-1$ in $G$.

The vertex $w$ partitions $\mathcal{F}$ into sets $\mathcal{F}(k)=\{G:|N(w) \cap S|=k\}$ where $0 \leq k \leq r$ if $w \notin S$ and $0 \leq k \leq r-1$ if $w \in S$.

For sets $R, T \subseteq V-v$ let $\mathcal{N}(R, T)=\mathcal{N}(R, T ; S, w)$ be the set of graphs in $\mathcal{F}$ with $N(w) \cap S=R$ and $N(w)-S=T$. If $|R|<|S-w|$, choose $x \in(S-w) \backslash R$ and $a \in T$. We consider a bipartite graph $\mathcal{B}$ with left vertex set $\mathcal{N}(R, T)$ and right vertex set $\mathcal{N}(R+x, T-a)$.

If $G \in \mathcal{N}(R, T)$ and $\{w, a\},\{x, b\}$ are edges of $G$ we make a switching $G:(w a, x b) \rightarrow$ $(w x, a b)$ in which edges $\{w, a\},\{x, b\}$ are replaced by $\{w, x\},\{a, b\}$ provided the resulting graph $G^{\prime}$ is simple. These switchings define the edges of $\mathcal{B}$, and $d_{L}(G)$ (resp. $\left.d_{R}\left(G^{\prime}\right)\right)$ is the number of edges incident with $G$ (resp. $\left.G^{\prime}\right)$ in $\mathcal{B}$.

Let $\nu(a, x ; G)=|N(a) \cap N(x)|$ be the number of common neighbours of $a$ and $x$ in $G$. Let $\delta(a, x ; G)=1$ if $a \in N(x)$.

Considering the possibilities for $b$ when the switching $G:(w a, x b) \rightarrow(w x, a b)$ gives a simple $G^{\prime}$ we have

$$
d_{L}(G)=|N(x)|-\nu(a, x ; G)-\delta(a, x ; G)
$$


for $G^{\prime}$ is simple iff $b \neq a$ and $b \notin N(a)$. Here $|N(x)|=r-1$ as $x \in S$. The switching leaves $\delta\left(a, x ; G^{\prime}\right)=\delta(a, x ; G)$ and $\nu\left(a, x ; G^{\prime}\right)=\nu(a, x ; G)$ as $(\{a\} \cup N(a)) \cap N(x)$ is the same set in both graphs.

Considering the switching $G^{\prime}:(w x, a b) \rightarrow(w a, x b)$ giving $G$ we have

$$
d_{R}\left(G^{\prime}\right)=|N(a)|-\nu\left(a, x ; G^{\prime}\right)-\delta\left(a, x ; G^{\prime}\right) .
$$

We note that $|N(a)|=r$ as $a \notin S$.

The graph $\mathcal{B}$ consists of components within which $\delta, \nu$ (and hence $d_{L}, d_{R}$ ) are invariant. Consider a component with bipartition size $\left(N_{L}, N_{R}\right)$. We now prove that $N_{L} \geq N_{R}$. In any component with edges we have $d_{R}=d_{L}+1$ so that $N_{R}=N_{L} d_{L} /\left(d_{L}+1\right)$. The case $\left(N_{L}, N_{R}\right)=(0,1)$ of isolated vertices in the right bipartition, cannot occur. For, in $G^{\prime}$

$$
\nu\left(a, x ; G^{\prime}\right)+\delta\left(a, x ; G^{\prime}\right) \leq|N(x)-w|=r-2
$$

and so

$$
d_{R}\left(G^{\prime}\right)=|N(a)|-\nu-\delta \geq 2 .
$$

Thus

$$
|\mathcal{N}(R, T)| \geq|\mathcal{N}(R+x, T-a)| \text {. }
$$

Given $S$ and $w$, the size of $\mathcal{N}(R, T ; S, w)$ is invariant for all $R, T,|R|=k$ by a simple symmetry argument.

Let $|\mathcal{N}(R, T ; S, w)|=\eta(k)$. Thus $\eta(k)$ is a non-increasing function of $k$. Let $f(k)=$ $|\mathcal{F}(k)|$ be the number of graphs in $\mathcal{F}$ with $|N(w) \cap S|=k$. If $w \notin S$ then for all $k \geq 0$, $f(k)=\left(\begin{array}{c}r \\ k\end{array}\right)\left(\begin{array}{c}n-2-r \\ r-k\end{array}\right) \eta(k)$. Similarly, if $w \in S$ then for all $k \geq 0, f(k)=\left(\begin{array}{c}r-1 \\ k\end{array}\right)\left(\begin{array}{c}n-1-r \\ r-1-k\end{array}\right) \eta(k)$.

Suppose $G$ is chosen u.a.r. from $\mathcal{F}(S)$ and let $Z(G)=|R|$. Then $\operatorname{Pr}(Z=k)=$ $f(k) /|\mathcal{F}|$. Writing $N=n-2, \rho=r-1_{w \in S}$,

$$
\operatorname{Pr}(Z=k)=\left(\begin{array}{l}
\rho \\
k
\end{array}\right)\left(\begin{array}{c}
N-\rho \\
\rho-k
\end{array}\right) \frac{\eta(k)}{|\mathcal{F}|} .
$$

Let $X$ be a hypergeometric random variable with $\operatorname{Pr}(X=k)=\left(\begin{array}{l}\rho \\ k\end{array}\right)\left(\begin{array}{c}N-\rho \\ \rho-k\end{array}\right) /\left(\begin{array}{l}N \\ \rho\end{array}\right)$. Then $\operatorname{Pr}(Z=k) / \operatorname{Pr}(X=k)$ decreases with $k$. It follows that $\operatorname{Pr}(Z \geq k) \leq \operatorname{Pr}(X \geq k)$ for any $k$.

The hypergeometric random variable $X$ has mean $\mu=\rho^{2} / N$. The proportional error in bounding $\operatorname{Pr}(X=j)$ above by $\operatorname{Pr}(Y=j)$, where $Y$ is the binomial random variable $B(\rho, \rho / N)$, is at most $\exp \left(\rho^{2} /(N-\rho)\right)$ (see [7] p57). Thus provided $r=o(\sqrt{n})$, using the following bound (2) on Binomial tails (see [1]),

$$
\operatorname{Pr}(Y \geq \beta \mu) \leq\left(\frac{e}{\beta}\right)^{\beta \mu}
$$


we see that

$$
\operatorname{Pr}(X \geq \beta \mu) \leq 2\left(\frac{e}{\beta}\right)^{\beta \mu}
$$

If $r \leq \log ^{2} n$ let $k=\alpha \rho+10, \alpha=1 / \log \log n$, then

$$
\operatorname{Pr}(X \geq \alpha \rho+10) \leq 2\left(\frac{e \rho^{2}}{(\alpha \rho+10)(n-2)}\right)^{\alpha \rho+10}=o\left(n^{-4}\right)
$$

For $\log ^{2} n \leq r \leq n$ let $k=\rho^{2} /(n-2)+4 \sqrt{\rho \log n}$. We can apply Azuma's inequality to the 0,1 sequence of observations of the sampling process of $X$, with $c_{i}=1$ to infer that

$$
\operatorname{Pr}\left(X \geq \rho^{2} /(n-2)+4 \sqrt{r \log n}\right)=o\left(n^{-4}\right) .
$$

Note that if $r \geq \log ^{2} n$ and $r=o(n)$ then the bound in (b) implies that in (a).

Lemma 4 Let $\mathcal{Q}_{2}$ be the event that no set of vertices $U \subset V$ of $G_{r}, 1 \leq|U| \leq n / 70$, induces more than $r|U| / 12$ edges. Then $\operatorname{Pr}\left(\mathcal{Q}_{2}\right)=1-O\left(1 / n^{2}\right)$.

Proof $\quad$ Let $\beta=1 / 12$ and $\theta=1 / 70$. Let $|U|=u$.

Note first that in a simple $r$-regular graph a set of size $u$ induces at most $\left(\begin{array}{l}u \\ 2\end{array}\right)$ edges and, provided $u \leq 2 \beta r$,

$$
\left(\begin{array}{l}
u \\
2
\end{array}\right) \leq \beta r u \text {. }
$$

Let $\mathcal{E}=\left\{F \in \Omega^{*}:\right.$ No vertex set $U, 2 \beta r \leq|U| \leq \theta n$ induces more than $\beta r|U|$ edges $\}$. It suffices to prove that $\operatorname{Pr}(\overline{\mathcal{E}})=O\left(n^{-2}\right)$.

In $\Omega$ the number of edges $X$ falling inside a set $U$ is dominated by a binomial random variable $Y \sim B(u r, u /(n-u))$ in which each configuration point of $U$ independently selects a pairing on the assumption that all configuration points of $U$ are available, and that $r u$ configuration points of $V \backslash U$ are unavailable. Now, $\mathbf{E} Y=r u^{2} /(n-u)$ and

$$
\begin{aligned}
\operatorname{Pr}_{\Omega}(Y \geq \beta r u) & =\operatorname{Pr}(Y \geq(\beta(n-u) / u) \mathbf{E} Y) \\
& \leq\left(\frac{u e}{\beta(n-u)}\right)^{\beta r u} \quad \text { by }(2) \\
& \leq\left(\frac{34 u}{n}\right)^{\beta r u} .
\end{aligned}
$$


As $r \geq 10^{6}, \beta r / 2 \gg 1$ and so by Lemma 2

$$
\begin{aligned}
\operatorname{Pr}(\overline{\mathcal{E}}) & \leq e^{2 r^{2}} \sum_{u=2 \beta r}^{\theta n}\left(\begin{array}{l}
n \\
u
\end{array}\right)\left(\frac{34 u}{n}\right)^{\beta r u} \leq e^{2 r^{2}} \sum_{u=2 \beta r}^{\theta n}\left(\frac{n e}{u}\right)^{u}\left(\frac{34 u}{n}\right)^{\beta r u} \\
& \leq e^{2 r^{2}} \sum_{u=2 \beta r}^{\theta n}\left(\frac{34 u}{n}\right)^{\beta r u / 2} \leq 2 e^{2 r^{2}}\left(\frac{68 \beta r}{n}\right)^{\beta^{2} r^{2}} \leq 2 \exp \left\{2 r^{2}-\beta^{2} r^{2} \log \frac{n}{6 r}\right\} \\
& =O\left(n^{-2}\right)
\end{aligned}
$$

provided $r \leq c_{0} n, c_{0}$ sufficiently small.

Proof of Theorem 1(a). Assume the events $\mathcal{Q}_{1}, \mathcal{Q}_{2}$ described in Lemmas 3,4. If $G_{r}$ is not $r$-connected then there is a separator $X$ of size $x \leq r-1$. Let $G_{r}-X=A+B$ and $|A|=a \leq|B|=b$.

Case 1: $2 \leq a \leq r / 2$.

Let $u, v \in A$ be arbitrary. If $r=o(n)$ then as $\mathcal{Q}_{1}$ occurs,

$$
|N(u) \cup N(v)| \geq 2 r-|N(u) \cap N(v)| \geq 2 r-o(r)-10
$$

However

$$
|N(u) \cup N(v)| \leq|A \cup X| \leq a+r-1<3 r / 2
$$

which contradicts $(3)$.

If $c n \leq r \leq n / 4$ for some $c>0$, we see that because $\mathcal{Q}_{1}$ occurs we have $|N(u) \cup N(v)| \geq$ $(1-o(1)) 7 r / 4$, which contradicts (4).

Case 2: $r / 2 \leq a \leq n / 80$.

As $|A \cup X| \leq a+r-1$ and $A \cup X$ contains at least $a r / 2$ edges we see that because $\mathcal{Q}_{2}$ occurs

$$
\frac{a r}{2} \leq \frac{r}{12}(a+r-1) \text { and so } a<r / 5 .
$$

Case 3: $n / 80 \leq a \leq\lceil n / 2\rceil$.

If configuration $F$ is chosen randomly from $\Omega$ then the existence of a separator of size $x \leq r-1$, where the smaller component has size $a \geq n / 80$, has probability at most

$$
\sum_{a=n / 80}^{\lceil n / 2\rceil} \sum_{x=0}^{r-1}\left(\begin{array}{l}
n \\
a
\end{array}\right)\left(\begin{array}{c}
n-a \\
x
\end{array}\right)\left(1-\frac{b}{n}\right)^{a r / 2} \text {. }
$$

Thus from Lemma 2 the probability of this event in $\mathcal{G}_{r}$ is at most

$$
e^{2 r^{2}} \sum_{a=n / 80}^{\lceil n / 2\rceil} 4^{n} e^{-a(n-(a+r)) r / 2 n} \leq e^{-r n / 500}=o(1)
$$

for $r \leq c_{0} n, c_{0}$ sufficiently small. 


\section{Hamilton cycles}

We prove Theorem $1(\mathrm{~b})$ on the assumption that $10^{7} \leq r \leq c_{0} n$.

Definition: Let $\mathcal{G}_{r}^{*}$ denote the subset of $\mathcal{G}_{r}$ consisting of those graphs $G$ with the following properties:

C1: All sets of vertices $U$ of size at most $n / 70$ induce at most $r|U| / 12$ edges.

C2: The graph $G$ is connected.

Lemma 4 and Theorem 1(a) imply that

Lemma $5\left|\mathcal{G}_{r}^{*}\right|=(1-o(1))\left|\mathcal{G}_{r}\right|$.

Given a subset $R$ of the edges of $G$, let $d_{R}(v)$ be the number of edges of $R$ which are incident with the vertex $v$ of $G$.

Definition: $\quad$ Let $P$ be some fixed longest path of $G$. A set of edges $R \subseteq E(G)$ is deletable from $G,(R \in \operatorname{Del}(G))$, if

D1: $R$ avoids $P$.

D2: For all $v \in V, \frac{r}{4} \leq d_{R}(v) \leq \frac{r}{2}$.

Lemma 6 Let $G \in \mathcal{G}_{r}$ and let $R$ be a random subset of the edges of $G$ where each edge of $G$ is placed into $R$ independently with probability $1 / 3$. then

$$
\operatorname{Pr}(R \text { is deletable } \mid G) \geq e^{-n}
$$

\section{Proof}

$$
\operatorname{Pr}(D 1 \mid G)=\left(\frac{2}{3}\right)^{|P|} \geq\left(\frac{2}{3}\right)^{n} \geq e^{-n / 2} .
$$

For (D2) we condition on (D1). We use the symmetric version of the Lovász Local Lemma (see for example Alon and Spencer [1]) to show that

$$
\operatorname{Pr}(D 2 \mid D 1) \geq e^{-n / 2} \text {. }
$$

Let $A_{v}$ be the event $\left\{d_{R}(v) \notin\left[\frac{r}{4}, \frac{r}{2}\right]\right\}$, then $\operatorname{Pr}\left(A_{v} \mid D 1\right) \leq e^{-r / 100}$ and the dependency graph has degree at most $r$. For large $r$ we can apply the lemma to show that conditional on $D_{1}$,

$$
\operatorname{Pr}\left(\bigcap_{v \in V} \overline{A_{v}} \mid D 1\right) \geq\left(1-2 e^{-r / 100}\right)^{n} \geq e^{-n / 2}
$$


The size of the set $R$ of deleted edges is binomial $B(r n / 2,1 / 3)$ and thus whp $|R|=(1+$ $o(1)) r n / 6$. For the purposes of Lemma 7 below, we condition on $|R| \in[(.16) r n,(.17) r n]$. We note that there exists some $\delta>10^{-7}$ such that

$$
\operatorname{Pr}(|R| \notin[(.16) r n,(.17) r n]) \leq e^{-\delta r n} .
$$

Definition: $\quad$ A set of edges $S$ is addable to a simple graph $H,(S \in \operatorname{Add}(H))$, if

A1: $H+S \in \mathcal{G}_{r}$.

A2: No longest path of $H$ is closed to a cycle by $S$.

Let

$$
\begin{aligned}
\mathcal{N} & =\left\{G \in \mathcal{G}_{r}^{*}: G \text { is not Hamiltonian }\right\} \\
\mathcal{E} & =\{(G, R): G \in \mathcal{N}, R \in \operatorname{Del}(G)\} \\
\Psi & =\{H: H=G-R,(G, R) \in \mathcal{E},|R| \in[(.16) r n,(.17) r n]\} \\
\mathcal{F} & =\left\{(G, S): G \in \mathcal{G}_{r}, G-S \in \Psi, S \in \operatorname{Add}(G-S)\right\} .
\end{aligned}
$$

Remark 4 We note that $\mathcal{E} \subseteq \mathcal{F}$ : Let $(G, R) \in \mathcal{E}$ so that $G-R \in \Psi$, and let $P$ be any longest path of $G$ avoided by $R$. By $(\mathrm{C} 2), G$ is connected, so $P$ cannot be contained in any cycle, as this would imply either that $G$ was Hamiltonian, or that $P$ was not a longest path. Thus $R$ is addable for $G-R$ and $(G, R) \in \mathcal{F}$.

Lemma 7 Let $H \in \Psi$. Let $\mathcal{S}(H)=\left\{S: H+S \in \mathcal{G}_{r}\right\}$. Let $S$ be chosen u.a.r from $\mathcal{S}(H)$. There exists a constant $\delta>10^{-7}$ such that

$$
\operatorname{Pr}(S \in \operatorname{Add}(H)) \leq e^{-\delta r n} .
$$

\section{Proof}

Given $y_{0}$ let $P_{y_{h}}=y_{0} y_{1} \ldots y_{h}$ be a longest path starting at $y_{0}$ in $H$. A Pósa rotation $P_{y_{h}} \rightarrow P_{y_{i+1}},[19,6]$ gives the path $P_{y_{i+1}}=y_{0} y_{1} \ldots y_{i} y_{h} y_{h-1} \ldots y_{i+1}$ formed from $P_{y_{h}}$ by adding the edge $y_{h} y_{i}$ and erasing the edge $y_{i} y_{i+1}$.

Let $\operatorname{END}(a)$ be any set of endpoint vertices formed by Pósa rotations with $a$ fixed, of a longest path $a P b$ in $H$. We prove that $|E N D(a)| \geq n / 210$.

The Pósa condition for the rotation endpoint set $U$ of a longest path $P$ requires that $|N(U)|<2|U|$, where $N(U)$ is the disjoint neighbour set of $U$. Let $u=|U|$ and let $\nu=|U \cup N(U)|$. Thus $u>\nu / 3$. The condition (D2) guarantees that $U \cup N(U)$ induces at least $r u / 4>r \nu / 12$ edges in $H$. Thus (C1) implies $\nu>n / 70$ and $u>n / 210$. 
Let the degree sequence of $R$ be $\mathbf{d}=\left(d_{1}, \ldots, d_{n}\right)$ and that of $H$ be $\left(r-d_{1}, \ldots, r-d_{n}\right)$. We choose a replacement set of edges $S$ of size $D=|R|=\left(d_{1}+d_{2}+\cdots+d_{n}\right) / 2$ uniformly among all edge sets with degree sequence $\mathbf{d}$ such that $H+S \in \mathcal{G}_{r}$. If we generate a random configuration $F$ on $\mathbf{d}$, then conditional on $H+\gamma(F)$ being simple, $\gamma(F)=S$ is a u.a.r element of $\mathcal{S}(H)$.

The probability that $H+\gamma(F)$ is simple.

We generate u.a.r. a configuration $F$ from the set $L$, size $|L|=2 D$, of configuration points corresponding to the degree sequence $\boldsymbol{d}$, of $R$. We show that

$$
\operatorname{Pr}(H+\gamma(F) \text { is simple }) \geq n^{-2} e^{-4 r^{2}} .
$$

We generate the first $r n / 12$ random pairings using CONSTRUCT and the rest of $F$ using GENERATE (see Remarks 2, 3). Our reason for this approach is as follows. The ordering $\boldsymbol{\sigma}=\left(x_{1}, x_{2}, \ldots, x_{2 D}\right)$ of $L$ in GENERATE is deterministic. At step $i=1$, the algorithm GENERATE defaults to Choice A. We cannot ignore the possibility that $H$ already contains the edge $\left\{\phi\left(x_{1}\right), \phi\left(x_{2}\right)\right\}$. Similarly, if at step $i+1$, GENERATE uses Choice B, then as the edges of $H$ are fixed, we cannot argue that the existing edges of $F_{i}$ avoid neighbours of $\phi\left(x_{1}\right), \phi\left(x_{2}\right)$ in $H$ until $i \gg r^{2}$.

Assuming that the $u_{i}$ are chosen randomly for each of the first $r n / 12$ iterations, we claim that the probability that CONSTRUCT inserts a loop or parallel edge is at most

$$
\frac{r / 2+r^{2} / 2}{(.15) r n} \leq 4 r / n \text {. }
$$

Indeed, when CONSTRUCT starts there are $2 D \in[(.32) r n,(.34) r n]$ configuration points to be paired. At the last iteration of CONSTRUCT there are $2 D-r n / 6 \geq(.15) r n$ points remaining. Each vertex occurs at most $r / 2$ times in the sequence (by D2).

CONSTRUCT picks a point $u_{i}$ and then a random point $v_{i}$. Given $u_{i}$ there are $\leq r / 2$ choices which make a loop. In the worst case $d\left(u_{i}\right)=r-1$ in $H+\gamma\left(F_{i-1}\right)$ and each neighbour is missing $r / 2$ points. This leads to at most $r / 2+r^{2} / 2$ bad choices out of at least (.15)rn choices for $v_{i}$.

Let $S_{1}$ be the subgraph of $S$ produced by CONSTRUCT. It follows that

$$
\operatorname{Pr}\left(H+S_{1} \text { is simple }\right) \geq e^{-r^{2}} .
$$

We now continue with GENERATE for the remaining $D-r n / 12$ edges to be inserted. The subgraph $H$ remains fixed, and GENERATE is initialized with configuration $F_{r n / 12}$ of $S_{1}$ on $\left\{u_{1}, u_{2}, \ldots, u_{r n / 6}\right\}$. For steps $i=r n / 12+1, \ldots, D$ we run GENERATE with the minimum degree ordering $\boldsymbol{\sigma}$ of $L-\left\{u_{1}, u_{2}, \ldots, u_{r n / 6}\right\}$ similar to the ordering described in the proof of Lemma 2. Observe that

$$
\operatorname{Pr}\left(H+\gamma\left(F_{i}\right) \text { is simple } \mid H+\gamma\left(F_{i-1}\right) \text { is simple }\right) \geq\left(1-\frac{1}{2 i-1}\right)\left(1-\frac{25 r}{n}\right) .
$$


The probability that the algorithm makes a Type B choice at step $i$ is $1-\frac{1}{2 i-1}$. Given a Type B choice, the probability that a loop or multiple edge is formed is at most $25 \mathrm{r} / n$ for reasons that we now explain. To create a loop we much choose $\phi\left(z_{t}\right)=\phi\left(x_{2 i+t-2}\right)$, for $t=1$ or 2 and there are at most $2 r$ choices of $\left\{z_{1}, z_{2}\right\}$ that will lead to this. To create a parallel edge $\phi\left(z_{t}\right)$ must be a neighbour of $\phi\left(x_{2 i+t-2}\right)$, for $t=1$ or 2 and there are at most $2 r^{2}$ choices of $\left\{z_{1}, z_{2}\right\}$ that will lead to this. These choices are made randomly from a set of edges of $F_{i}$ of size at least $r n / 12$.

Now $\prod_{i=r n / 12+1}^{D}\left(1-\frac{1}{2 i-1}\right) \geq n^{-2}$. The number of edges inserted by GENERATE is at most (.087)rn and $\left(1-\frac{25 r}{n}\right)^{(.087) r n} \geq e^{-3 r^{2}}$ and so (7) follows.

The probability that $\gamma(F)$ is addable for $H$.

Let $x_{0}$ be an end vertex of longest path $P$ in $H$. Now let $Y=\{(a, b): a \in$ $\left.E N D\left(x_{0}\right), b \in E N D(a)\right\}$. Then $S \in A d d(H)$ implies $\gamma(F) \cap Y=\emptyset$. For otherwise the edge $a b$ would close some longest path of $H$ to a cycle.

We will use CONSTRUCT to generate a configuration $F$ with the required degree sequence $\left(d_{1}, \ldots, d_{n}\right)$.

Since $\left|E N D\left(x_{0}\right)\right| \geq n / 210$, the sum of the values $d_{v}$ over vertices $v \in E N D\left(x_{0}\right)$ is at least $\frac{r}{4} \frac{n}{210}$. Thus, we can choose $u_{j}$ so that $\phi\left(u_{j}\right) \in E N D\left(x_{0}\right)$ for each of the first $\nu=r n / 1680$ steps. For $j \leq \nu$, writing $a$ for $\phi\left(u_{j}\right)$, let $Y_{j}$ be the set of remaining configuration points $y$ such that $\phi(y) \in E N D(a)$. Then $\left|Y_{j}\right| \geq \frac{r}{4} \frac{n}{210}-2 j$. As $F$ contains at most $r n / 2$ configuration points,

$$
\begin{aligned}
\operatorname{Pr}(\gamma(F) \cap Y=\emptyset) & \leq \prod_{j=1}^{\nu}\left(1-\frac{\left|Y_{j}\right|}{r n / 2}\right) \\
& \leq \exp \left(-\sum_{j=1}^{\nu}\left(\frac{1}{420}-\frac{4 j}{r n}\right)\right) \\
& =e^{-\delta_{1} r n}
\end{aligned}
$$

where $\delta_{1} \approx 1 /(1680 \times 840)$.

Thus

$$
\operatorname{Pr}(S \in \operatorname{Add}(H)) \leq e^{-\delta_{1} r n} \times n^{2} e^{4 r^{2}}
$$

and the lemma follows.

We can now complete the proof of Theorem 1(b). Suppose $G$ is chosen u.a.r. from $\mathcal{G}_{r}^{*}$ and then $R$ is chosen by selecting edges independently with probability $1 / 3$. From 
Lemma 6, we see that

$$
\begin{aligned}
\operatorname{Pr}(\mathcal{E}) & =\sum_{G \in \mathcal{N}} \sum_{R \in \operatorname{Del}(G)} \operatorname{Pr}((G, R)) \\
& \geq e^{-n} \operatorname{Pr}(\mathcal{N}) .
\end{aligned}
$$

From the definitions (6), inequality (5) and Lemma 7 it follows that

$$
\begin{aligned}
\operatorname{Pr}(\mathcal{F}) & \leq \operatorname{Pr}(|R| \notin[(.16) r n,(.17) r n]) \\
& +\sum_{H \in \Psi} \sum_{S \in A d d(H)} \operatorname{Pr}((H+S, S) \mid G-R=H) \operatorname{Pr}(G-R=H) \\
& \leq \sum_{H \in \Psi} e^{-\delta r n} \operatorname{Pr}(G-R=H)+e^{-\delta r n} \\
& \leq 2 e^{-\delta r n}
\end{aligned}
$$

Now, by $\operatorname{Remark} 4, \mathcal{E} \subseteq \mathcal{F}$ and so $\operatorname{Pr}(\mathcal{E}) \leq \operatorname{Pr}(\mathcal{F})$, thus

$$
\operatorname{Pr}(\mathcal{N}) \leq 2 e^{n-\delta r n}=o(1)
$$

and the theorem follows from Lemma 5.

Remark 5 We note that by following Frieze [10] we can, at the expense of complicating the proof, prove the existence of a polynomial time algorithm for finding a Hamilton cycle.

Acknowledgement: We wish to thank an anonymous referee for several very careful and thorough reviews, which contributed greatly to the clarity of exposition and accuracy of the paper.

\section{References}

[1] N.Alon and J.Spencer, The Probabilistic Method, Wiley, 1992.

[2] E.A.Bender and E.R.Canfield, The asymptotic number of labelled graphs with given degree sequences, Journal of Combinatorial Theory, Series A 24 (1978) 296-307.

[3] B.Bollobás, A probabilistic proof of an asymptotic formula for the number of labelled regular graphs, European Journal on Combinatorics 1 (1980) 311-316.

[4] B.Bollobás, Random Graphs, in Combinatorics (ed. H.N.V.Temperley), London Mathematical Society Lecture Note Series 52, Cambridge University Press, Cambridge (1981) 80-102. 
[5] B.Bollobás, Almost all regular graphs are Hamiltonian, European Journal on Combinatorics 4, (1983) 97-106.

[6] B.Bollobás, Random Graphs, Academic Press, 1985.

[7] W. Feller. An Introduction to Probability Theory. Volume I, Wiley (1960).

[8] T.I.Fenner and A.M.Frieze, Hamiltonian cycles in random regular graphs, Journal of Combinatorial Theory, Series B 37 (1984) 103-112.

[9] A.M.Frieze, Random regular graphs of non-constant degree, Technical Report, Department of Mathematical Sciences, Carnegie Mellon University (1988).

[10] A.M.Frieze, Finding Hamilton cycles in sparse random graphs, Journal of Combinatorial Theory Series B 44 (1988) 230-250.

[11] A.M.Frieze and T.Euczak, On the independence and chromatic numbers of random regular graphs, Journal of Combinatorial Theory Series B 54 (1992) 123-132.

[12] S.Janson, T.Łuczak and A.Ruciński, Random Graphs, John Wiley and Sons, 2000.

[13] M.Krivelevich, B.Sudakov, V.Vu and N.C.Wormald, Random regular graphs of high degree, to appear in Random Structures and Algorithms.

[14] L.Kučera, Canonical labelling of regular graphs in linear expected time, Proceedings of the 28th Annual IEEE Symposium on Foundations of Computer Science (1987) 271-279.

[15] T.Łuczak, Sparse random graphs with a given degree sequence, in Random Graphs Vol. 2, (eds. A.M.Frieze and T.Łuczak), Wiley, New York (1992) 165-182.

[16] B.D.Mckay, Asymptotics for symmetric 0-1 matrices with prescribed row sums, Ars Combinatoria 19A (1985) 15-25.

[17] B.D.Mckay and N.C.Wormald, Asymptotic enumeration by degree sequence of graphs with degree o $\left(n^{1 / 2}\right)$, Combinatorica 11 (1991) 369-382.

[18] B.D.Mckay and N.C.Wormald, Asymptotic enumeration by degree sequence of graphs of high degree, European Journal of Combinatorics 11 (1990) 565-580.

[19] L.Pósa, Hamilton circuits in random graphs, Discrete Mathematics 14 (1976) 359364.

[20] R.W.Robinson and N.C.Wormald, Almost all cubic graphs are Hamiltonian, Random Structures and Algorithms 3 (1992) 117-126.

[21] R.W.Robinson and N.C.Wormald, Almost all regular graphs are Hamiltonian, Random Structures and Algorithms 5 (1994) 363-374. 
[22] N.C.Wormald, Models of random regular graphs, Surveys in Combinatorics, London Mathematical Society Lecture Note Series 267, Cambridge University Press, Cambridge, 1999 (J.D.Lamb and D.A.Preece, Eds.), Proceedings of the 1999 British Combinatorial Conference, Cambridge University Press, 239-298.

This research was sponsored in part by National Science Foundation (NSF) grant no. CCR-0122581. 